\title{
Vaccination Hesitancy On Introduction Of COVID 19 Vaccine In Pakistan: A Web-Based Cross Sectional Study April 2021
}

\author{
Sobiya Sawani ( $\square$ sobiya.sawani@aku.edu ) \\ Aga Khan University Hospital \\ Rehana Siddiqui \\ Aga Khan University Hospital \\ Bilal Ahmed Usmani \\ Aga Khan University Hospital \\ Syed lqbal Azam \\ Aga Khan University Hospital \\ Durr E Amna Siddiqui \\ Aga Khan University Hospital \\ Hajra Khwaja \\ Aga Khan University Hospital \\ Sehrish Karim \\ Aga Khan University Hospital
}

\section{Research Article}

Keywords: Vaccine Hesitancy, COVID-19, vaccine safety, health services, survey

Posted Date: February 24th, 2022

DOl: https://doi.org/10.21203/rs.3.rs-1358762/v1

License: (9) (i) This work is licensed under a Creative Commons Attribution 4.0 International License. Read Full License 


\section{Abstract}

Background: Vaccination against COVID-19 is one of the control measures for an ongoing COVID-19 pandemic. Vaccine acceptance is known to be low in Pakistani population, we identified the factors associated with vaccine hesitancy (VH) for COVID-19 at the time of first introduction of this vaccine for elderly and health care workers.

Methods: An online survey was launched using a google form during March to April 2021 to assess VH in adult (> 18 years) Pakistani population. The survey form determined VH individuals who declined to questions on getting vaccinated and maintained a perspective of reluctance to vaccinate. In addition to sociodemographic characteristics, observation of standard precautions, and sources of information on COVID-19 vaccine were inquired.

Results: Of the 278 responses $80 \%$ of the study population was less than 45 years of age, $68 \%$ were males, $91 \%$ had 16 or more years of education, $39 \%$ were in health care profession, $81 \%$ reported consistent use of face masks in public places, $68 \%$ sanitized hands each time, $35 \%$ always avoided social gatherings, $24 \%$ had received vaccination, $20 \%$ had suffered from COVID19 , and $45.7 \%$ were vaccine hesitant. At multivariable level, being health care worker, increasing mean precautionary score, not being vaccinated against COVID-19 and those with concerns for vaccine safety were the contributing factors for VH. Participants who presumed COVID-19 vaccine was safe were $90 \%$ less hesitant to get COVID-19 vaccine (Adjusted Prevalence Ratio: 0.1; 95\% $\mathrm{Cl} 0.09,0.29)$ than who were uncertain on vaccine safety.

Conclusion: Reliable safety information on COVID-19 vaccine and trust building influenced VH, to mass vaccinate Pakistani population to control the pandemic.

\section{Background:}

Vaccine against COVID-19 since its introduction has been a key strategy to contain the pandemic and avoid adversities associated with the disease (1). The development of vaccine is a complex and time-consuming process which takes around 15 years and more, starting with designing and exploratory preclinical work later followed by multiple phases in which formal preclinical experiments and toxicology studies are conducted (2). COVID-19 vaccine development has significantly reduced this timeline to 12 to 24 months by omitting some of the initial steps of the exploratory phase (2). In early 2020extensive efforts were made for COVID-19 vaccine development by research centers, pharmaceutical companies, and institutions around the world (3). Moderna Inc initiated the first trial in USA in March 2020 (4). Around 160 vaccines were already into development globally (5). In January 2021, Pfizer and Moderna vaccines were available in the USA; Oxford University vaccine was made available in UK and Sinovac in China (6). Due to limited supply, health care workers (HCWs) were the first recipients of these vaccines followed by people of age 60 years and above. While development of COVID-19 vaccine itself is a challenge, convincing the target population is another big problem, especially for country like Pakistan which is struggling to eradicate diseases such as polio and measles due to vaccine resistance (7-8). Hesitancy to COVID-19 vaccine was a challenge in early 2020's that might create obstacles in acquiring the required immunity $(9-10)$.

According to WHO, Strategic advisory group of experts (SAGE), vaccine hesitancy is defined as "delay in acceptance or refusal of vaccination despite [the] availability of vaccine services" (11). The vaccine refusal and delays are the root cause to an increased number of vaccine preventable diseases and is also regarded as one of the threats to global health by WHO. There is a widespread mistrust among public towards companies who produce vaccines, health system who are involved in development and distribution of vaccines, healthcare workers who deliver the vaccines and perception towards vaccine safety, effectiveness, and its importance. False religious beliefs and misleading information by influential leaders, social media, friends, and family are significant contributor to vaccine refusals, creating fear and uncertainty around vaccines (12-14). According to a systematic review, low rates of COVID-19 vaccine acceptance were reported by Middle East (26.6), Russia ((54.9\%) and several European countries (58.9\%) (15). Reported factors associated with both refusal and delay to covid-19 vaccination, includes being old, female gender, low education, less confidence in the COVID-19 vaccine, source of information, being a HCW in general, working in the specialty of medicine and allied, taking direct care of COVID-19 patients, and those who had a prior COVID-19 infection (8, 16-17).

Page 2/12 
Pakistan is facing multiple challenges in achieving COVID-19 immunity threshold via vaccine administration in the country. The issues range from mistrust, lack of education, strong influence of religious leaders, false propaganda, and emergence of new variants. In addition to past exposure to the virus, high vaccination rates are required to achieve $60-90 \%$ threshold for herd immunity, by effectively interrupting the transmission chain. Pakistan achieved the target for more than $40 \%$ of the population vaccinated by the end of 2021 (18). To date (25th Jan 2022), around 1.7 billion doses have been administered, and more than 80 million people are fully vaccinated, corresponding to 36 percent of the Pakistani population (19).

\section{Rationale}

COVID-19 vaccine is a beacon of hope for return to the usual routine lifestyle prior to COVID-19 pandemic but undermined by VH. Unfortunately, non-vaccinated and under-vaccination remains problematic in many parts of the world.

COVID-19 vaccination is facing a significant challenge in achieving the vaccination coverage required for population immunity. Findings from studies suggest that populations negative attitude and unwillingness to receive the vaccine due to parental refusal, conspiracies and myths, misconceptions, concerns about its safety and its effectiveness are the reason for VH (20-21). In addition, factors like age, gender, socio-economic background, region of residence and knowledge about vaccine is also playing a role in acceptance (22). Studies from Pakistan indicate that $\cong 25 \%$ were vaccine hesitant (8). Pakistan is expected to face significant $\mathrm{VH}$ publicly given the current estimates and population behaviors and concerns for other vaccine preventable diseases. Such hesitancy about the vaccination regardless of availability poses a major challenge when it comes to successful inoculation. Identifying the factors for under-vaccination or non-vaccination is important in any given community especially in times of dire need, will provide clarity about the practices and concerns of the population. Therefore, we assessed the $\mathrm{VH}$ among adult population in early parts of the year 2021, when vaccination against COVID-19 was initiated, identifying the associated factors of VH. This will help in establishment of better immunization strategies for increasing the demand in unvaccinated people, preventing the unnecessary sufferings from vaccine preventable disease.

\section{Objectives}

1. To assess the COVID-19 vaccine hesitancy in adult Pakistani population.

2. To identify the factors that affect vaccine hesitancy in the adult Pakistani population.

\section{Methodology:}

\section{Study design and Study participants}

A web based cross-sectional study design was employed to assess VH for COVID-19 vaccination among the adult population of Pakistan in April 2021, when the third wave of the pandemic was emerging. This study enrolled males and females, above 18 years of age, living in Pakistan, through social media platforms using a self-administered tool in English language.

\section{Study duration and Sample size}

Pakistan received the 1 st consignment of Sinopharm with 500,000 doses from China on 1 st Feb 2021 \& the vaccination process began in the country on 3rd Feb 2021 where HCW being the first recipients of the vaccine. Later from March 10th, 2021 the vaccination process also started for 60 years and above group. A minimum sample size of 278 participants was considered to achieve study objectives anticipating the range of VH from 10-45\% (16). The data collection for this study took two-weeks' time from April 1 to April 14th, 2021.

\section{Data Collection Procedure and tool}

Data were collected using structured questionnaire created in English language as a Google Form, that was shared on various social media sites (e.g Facebook, what's app, Twitter) and networks such as academic posts, community organizations, personal groups, and in-person requests. 
The questionnaire had three sections. The first section contained basic socio-demographic and employment information. The second section inquired on information related to COVID-19 disease, which included history of infection and practices of study participants for precautionary measures, and the third section explored information on COVID-19 vaccine and VH as the main outcome variable. The structured questionnaire was available to participants in English language taking approximately 10 minutes of their time to complete.

Vaccine hesitant individual was the one who responded "No" to the question "Would you like to get vaccinated against Covid-19 infection" (scale of 1 as a definitely not to 5 as definitely) and how would you describe your attitude towards receiving a COVID19 vaccine (1- Against the vaccine to 5 - Very keen to vaccinate). These items were rated on a scale of $1-5$. The above items were then converted into binary variable as "Yes" from response of 4-5\& "No" from response of 1-3.

A variable for scoring practices was created from information collected in second section for observing precautionary measures; reporting on wearing of masks at public places, hand sanitization, and observing social distancing. Each category was given score of $0-3$; the higher score of 3 for each category meant a better precautionary practice. The grand total of these items ranged between $0-9$. The questions in third sections on vaccine uptake, safety and reliability, side effects related to vaccination, reasons for refusal, and sources of information for COVID-19 vaccination.

\section{Data Analysis}

Frequencies and percentage were reported for age groups, gender, province, marital status, level of education, profession, COVID19 disease, and vaccine related information. Mean and SD was reported for age. Prevalence Ratio (PR) using cox proportion algorithm was employed to determine the COVID-19 VH in adult population. Univariate and multivariable analyses were done using Cox hazard algorithm. The results are reported as crude and adjusted prevalence ratio (APR) with $95 \%$ confidence interval. A p-value of less than 0.05 was considered statistically significant. For analysis of the data, STATA version 15 was used.

\section{Results:}

Overall, 278 respondents answered the online survey, of which 127 (45.7\%; $95 \%$ Cl: 39.8\%, 51.5\%) were vaccine hesitant at the time of this study. The mean age of the study participants were $34.2(\mathrm{SD}=12.7)$ years, and $189(67.9 \%)$ were females. (Table 1). The majority of the responses of $77 \%$ were received from Sindh province, $11 \%$ from Punjab and rest (12\%) from other provinces and regions. Majority (91\%) of the participants in the study were either graduates or postgraduates and $39.1 \%$ were the HCW. About one fifth of the study participants had a history of COVID-19 disease before this survey $(20.9 \% ; 95 \%$ Cl: 16.1 , $25.6 \%)$.

More than $91.3 \%$ of the participants in the study agreed that complications associated with COVID-19 are serious. For variables taking COVID-19 precautionary measures, like wearing mask at public and workplace was reported to be practiced by $81 \%$ whereas few study participants (35.1\%) avoided social gatherings. The mean precautionary score was found to be 7.5 (SD: 1.4).

Table 1: Socio-demographic characteristics \& COVID-19 disease information of the study participants $(\mathrm{n}=278)$ 


\begin{tabular}{|c|c|c|}
\hline Characteristics & $\begin{array}{l}\text { Number } \\
(n=278)\end{array}$ & $\begin{array}{l}\text { Percentage } \\
\text { (\%) }\end{array}$ \\
\hline \multicolumn{3}{|l|}{ Age in years } \\
\hline $18-24$ & 57 & 20.5 \\
\hline $25-44$ & 166 & 59.7 \\
\hline $45-59$ & 39 & 14.0 \\
\hline $60+$ & 16 & 5.8 \\
\hline \multicolumn{3}{|l|}{ Gender } \\
\hline Male & 89 & 32.1 \\
\hline Female & 189 & 67.9 \\
\hline \multicolumn{3}{|l|}{ Level of Education } \\
\hline$\leq$ Inter/A-level & 13 & 4.7 \\
\hline Graduate & 122 & 43.7 \\
\hline Post-graduate & 132 & 47.4 \\
\hline \multicolumn{3}{|l|}{ Marital Status } \\
\hline Single & 107 & 38.5 \\
\hline Married/Divorced/Widowed & 171 & 61.5 \\
\hline \multicolumn{3}{|l|}{ Health Care Provide } \\
\hline Yes & 109 & 39.1 \\
\hline No & 169 & 60.6 \\
\hline \multicolumn{3}{|l|}{ History of COVID-19 infection } \\
\hline Yes & 58 & 20.8 \\
\hline No & 220 & 78.9 \\
\hline \multicolumn{3}{|c|}{ Participants opinion; if complications from Covid-19 are serious } \\
\hline Strongly agree & 172 & 61.6 \\
\hline Agree & 83 & 29.7 \\
\hline No Opinion & 16 & 5.7 \\
\hline Disagree & 7 & 2.52 \\
\hline \multicolumn{3}{|c|}{ Observing SOP's of wearing face mask public/workplace } \\
\hline Every time & 226 & 81.2 \\
\hline Sometime & 38 & 13.6 \\
\hline Often & 14 & 5.1 \\
\hline Never & 0 & 0 \\
\hline \multicolumn{3}{|c|}{ Observing SOP's of using hand sanitization } \\
\hline Every time & 190 & 68.3 \\
\hline Sometime & 66 & 23.7 \\
\hline
\end{tabular}

Page 5/12 


\begin{tabular}{|lcc|}
\hline Often & 19 & 6.8 \\
\hline Never & 3 & 1.1 \\
\hline Observing SOP's of avoiding social gatherings & \\
\hline Yes, always & 98 & 35.1 \\
\hline Sometimes & 126 & 45.2 \\
\hline Occasionally avoid/ Do not avoid at all & 54 & 19.4 \\
\hline
\end{tabular}

About 1/4th (24.1\%; $95 \% \mathrm{Cl}: 19.1 \%, 29.1 \%)$ of the study participants were already vaccinated for COVID-19 with at least one dose. Among those who were vaccinated $57.0 \%$ (95\% Cl: $47.6 \%, 66.2 \%)$ were HCW's and only 3.0\% (95\% Cl: $4.0 \% 5.5 \%)$ were non HCW's.

One third of the study participants reported for being worried about getting some major side effect after COVID-19 vaccine like blood clotting, breathing problems, getting COVID-19 disease, mental and physical health issues, organ damage and death. Thirty percent reported to be fearful for side effects like fever, headache, allergies, pain and fatigue. Few participants either did not know about the side effects (23.4\%) or reported experience any side effect (13.3\%) from COVID-19 vaccine. Highest (44.8\%) VH was identified among those who reported that vaccine is associated with some major side effect and lowest (14.9\%) was seen among people who reported that there are no side effects related to COVID-19 vaccine (Fig 1a). More than half (51.1\%) of the study participants reported social media as the source of COVID-19 vaccine information, $26.9 \%$ relied on government website, 19.4 on academic source and few $2.5 \%$ did not know which source should be trusted. VH was reported highest (55.1\%) among those who relied on social media for COVID-19 vaccine information and lowest (4.7\%) among individual who did not know which source to be considered reliable (Fig 1b). The reason of refusal for COVID-19 vaccine was assessed among all study participants, $66.5 \%$ had trust issues for the new vaccine where very few only $1.1 \%$ had no issue in getting COVID-19 vaccine (Fig 1c). In this study majority $(76.2 \%)$ of study participants were willing to get vaccination if recommended by their physicians. $45.3 \%$ were also willing to pay for getting COVID-19 vaccine where more than half (54.6\%) did not agree or were not sure if they want to pay for getting the vaccination. Among vaccine hesitant $75.2 \%$ of individuals were willing to encourage their family and friends for COVID-19 vaccine dose. This study also identified the reasons of hindrance for COVID-19 vaccine beside willingness (Fig 1d).

Figure 1: Vaccine Hesitancy among those (a) who were concerned about types of side effects, (b) reliable sources of COVID-19 vaccine information, (c) reasons for refusal for COVID-19 vaccination \& (d) reasons for hindrance for COVID-19 vaccination besides having willingness

At univariate level, age, marital status, being health care provider, increasing mean precautionary scores, being vaccinated, its side effect and opinion about COVID-19 vaccine were the variables eligible for multivariable analysis. VH decreased with increase in age ( $P$-value: 0.049). Participants with marital status as single were more vaccine hesitant than ever married ( $P$ value: 0.195$)$, being health care providers ( $P$-value: 0.005$)$ and those practicing precautionary measure ( $P$-value: 0.001$)$ were less vaccine hesitant. In this study, participant who were not vaccinated against COVID-19 ( $P$-value: $<0.00 I)$ and who reported some major side effects related to vaccination ( $P$-value: 0.003$)$ were more vaccine hesitant. Study participants who thought COVID-19 was a trustworthy vaccine $(P$-value: $<0.00 l)$ were $90 \%$ less vaccine hesitant compared to participants who were unsure. Similarly, participants who thought COVID-19 was safe vaccine ( $P$-value: $<0.00$ l) were $80 \%$ less hesitant to COVID-19 vaccine as compared to those who thought vaccine was not safe/ unsure.

At multivariable level being health care worker, increasing mean precautionary score, being already vaccinated against COVID-19 and considering COVID-19 vaccine was safe were the contributing factors. Study participants who thought COVID-19 vaccine was safe were $90 \%$ less hesitant to COVID-19 vaccine as compared to those who thought vaccine was not safe/ unsure (APR: $0.1 ; 95 \% \mathrm{Cl} 0.09,0.29$ ) when controlling for variables in the final model. (Table: 2 ).

Table 2: Determinants of Vaccine Hesitancy among study participants $(n=278)$ 


\begin{tabular}{|c|c|c|}
\hline Characteristics & Crude PR $(95 \% \mathrm{Cl})$ & Adjusted PR(95\% Cl) \\
\hline Age (Years) & $0.9(0.96,1.0)$ & \\
\hline \multicolumn{3}{|l|}{ Marital Status } \\
\hline Single & $1.2(0.88,1.78)$ & \\
\hline Married/Divorced/Widowed (Ref) & - & \\
\hline \multicolumn{3}{|l|}{ Health care worker } \\
\hline Yes & $0.6(0.38,0.83)$ & $1.2(0.83,1.97)$ \\
\hline \multicolumn{3}{|l|}{ No (Ref) } \\
\hline Mean precautionary score & $0.8(0.74,0.92)$ & $0.9(0.82,1.05)$ \\
\hline \multicolumn{3}{|l|}{ Vaccinated for COVID-19 } \\
\hline \multicolumn{3}{|l|}{ Yes (Ref) } \\
\hline No & $5.4(2.54,11.66)$ & $3.3(1.45,7.66)$ \\
\hline \multicolumn{3}{|l|}{ COVID-19 side effects } \\
\hline Major & $2.1(1.24,3.52)$ & \\
\hline Minor & $1.1(0.64,2.06)$ & \\
\hline Don't know & $2.1(1.15,3.90)$ & \\
\hline None (Ref) & - & \\
\hline \multicolumn{3}{|l|}{ COVID-19 vaccine is trustworthy } \\
\hline Yes & $0.1(0.07,0.23)$ & \\
\hline No & $1.2(0.76,1.94)$ & \\
\hline Maybe /not sure /Don't Know (Ref) & - & \\
\hline \multicolumn{3}{|l|}{ COVID-19 vaccine is safe } \\
\hline Yes & $0.2(0.67,0.37)$ & $0.1(0.13,0.68)$ \\
\hline No & $1.1(0.72,1.96)$ & $1.0(0.66,1.71)$ \\
\hline Maybe /not sure /Don't Know (Ref) & - & - \\
\hline
\end{tabular}

\section{Discussion:}

The $\mathrm{VH}$ rate during the first two weeks of April 2021 was 45.7\%, in a relatively educated study participants than a Pakistani general population. Studies conducted in early phases of the pandemic between March to April 2020 showed a high reception, above $90 \%$, for potential vaccine acceptance in China, Malaysia and Indonesia $(3,23)$. Studies conducted in later phases of the pandemic from October to December 2020 showed a declining trend in acceptance rate in America, Jordan and Kuwait at 66.8\%, $30.9 \%$ respectively, with Kuwait acceptance rate standing at only $29.4 \%(24-25)$. Studies conducted during vaccine rollout stage, from 2021 onwards, showed a 31.1\% hesitancy rate in Italy in January 2021 when HCW had started receiving vaccinations. (26) In Japan in April 2021, almost $60 \%$ of the population was either unsure or in complete refusal to receive the vaccine. (27)

A study from Pakistan, that took place from December 2020 to March 2021, reported the VH of 35.8\% that is less than the VH rate of this study (45.7\%) (28). Whereas in comparison to a study done from January to March 2021 , it was slightly lower than the reported $\mathrm{VH}$ rate of $47 \%$ (29). The reason for this could be the changing perceptions of studied population overtime, with $\mathrm{VH}$ rates increasing as the act of receiving a vaccine became reality and people's fears surfaced. These findings are in line with a 
longitudinal study done within the US to see changes in Covid 19 vaccine acceptance; their findings showed a decreasing trend $\mathrm{VH}$ rates over 6 months, which could be attributed to information sources (30). A similar trend was seen in China with acceptance declining over time (31).

Our study was carried out in the initial days of vaccine distribution when allowance was only given to healthcare workers and citizens over 60 years of age; and the mean age of the study participants was 34.2 with majority (59.7\%) being between the age of 25-44. This shows that most participants were not eligible to receive the vaccine so those who were, were more open to it. This is reflected in our study as well, the VH decreased with inappcreasing age ( $P$-value: 0.049). This finding is concurrent with studies done in both China (31) and Canada (32) finding older individuals extremely willing to receive the vaccine.

Within the vaccinated population, majority (57\%) were health care workers (HCW), however, among HCWs more than one third $(31.2 \%)$ were still unwilling to receive the vaccine. A positive correlation was found between being a health care worker and hesitance to the vaccine after adjusting the PR $1.2(0.83,1.97)$. Studies done on vaccine acceptance rates in HCW in different countries are rare and show a wide range in rates; this disparity could be owed to cultural, political or religious differences. Data supporting our study has similarly appeared from Saudi Arabia and Palestine with VH in HCWS at $49.48 \%$ and $30.7 \%$ respectively (33-34). HCW were the first priority of WHO when vaccine resources were a concern as they serve as the first line to be affected and transmit the virus. This could have been a factor as receiving vaccine before others might have made the health care workers cautious to vaccination. In the study in Palestinian HCW vagueness of the efficacy and side effects were cited as the main cause of hesitancy (34).

Skepticism for the vaccine was determined through vaccine safety and vaccine trustworthiness. Majority (66\%) had trouble trusting the vaccine along with a positive correlation (adjusted PR value of 1.0) between thinking the Covid vaccine is not safe and therefore were $\mathrm{VH}$. This could be due to this study being carried out in the early phase of rollout, the speed of vaccine development and fake news circulation which is why reassurance and guidance by physicians was cited as an important factor to increase vaccine acceptance $(76.2 \%)$. Over $60 \%$ of participants were worried about major side effects of the vaccine ranging from breathing problems, blood clotting, getting Covid 19, to death. A very small minority (23.4\%) was unaware of the side effects from the vaccine. A major reasoning for differences in opinions could be the sources where people are getting their information as majority (51\%) reported to get information from social media where sources are not fact checked and can lead to a snowball effect of escalating rumors (35). Not surprisingly, $\mathrm{VH}$ was most consistent in those believing that vaccine had major side effects (44.8\%).

According to the Health Belief Model (HBM), perceived severity includes believing that the disease in question can cause serious harmful effects and according to our survey, most of the participants did believe that Covid 19 resulted in serious complications (36). This is complemented by the fact that the precautionary score calculated within our study was 7.5 and more than three quarter of participants complied to wearing masks in public and majority made use of hand sanitizers. Despite this, more than $60 \%$ of participants were concerned about the effectiveness of the vaccine in the first place. With the progression of the pandemic, people might have become more reliant on and comfortable with measures being taken already such as mask wearing, hand washing and sanitizers with the belief that such precautions are sufficient (31).

The belief that actively getting the disease or disease severity can be reduced by getting the vaccine was not assessed. This serves as a limitation for assessment of risk perception which is an important indicator within epidemiology to control infectious disease epidemics (34). Most of our participants were part of the educated strata who had both access to the internet plus basic skills to fill out the questionnaire in English language, hence the findings from our study cannot be generalized to the whole population as Pakistan has a literacy rate of $59 \%$ only and majority of the population don't speak English language. Due to constraints of observing standard procedures during the pandemic our study had to be conducted via online surveys which could have decreased sample size and any information and reporting bias as there is no way of ensuring quality of collected information. Another issue is the timeline of our study, which can only capture the attitude at that particular moment, and perhaps with initiation of month of Ramadan. However, as time proceeds, new variants cropping up along with changes in policies and restrictions, the attitude towards vaccines could keep changing so further studies particularly those inculcating the HBM are needed. Despite all these limitations, we are confident that findings from our study could make way for better policies 
in Pakistan targeting towards increasing vaccine awareness through health education interventions, mitigating false propaganda related to vaccine on social media, clinician recommendations for COVID-19 vaccine to their patients and educate masses about the safety and effectiveness of COVID-19 vaccine. The vaccine trials and public release of safety and efficacy information and trust building activities could also reduce COVID-19 VH in our setting.

Our data suggests that VH related to COVID-19 is of utmost concern and needs serious action by the policy on communicating by government to address this issue. Pakistan has had consistent trepidations towards vaccines even before Covid- 19 hit. Pakistan is among the countries - Pakistan, Afghanistan and Nigeria where distrust in vaccines will increase over the year as religious extremism prevails here along with civil uncertainty. This leads to spreading of false information and propaganda, especially if one considers polio cases are still existent here (12). In order for Pakistan to overcome this hurdle the first step needs to be probing research on the relationship between political and religious polarity and vaccination beliefs.

\section{Abbreviations:}

VH: Vaccine Hesitancy; HCWs: health care workers; WHO: World Health Organization; SAGE: Strategic advisory group of experts; PR: Prevalence Ratio; APR: Adjusted prevalence ratio; SOP: Standard operating procedure.

\section{Declarations:}

\section{Ethics approval and consent of participate}

Ethical approval for this study has been obtained from the Aga Khan University Ethical Review Committee (approval number: 2021-6125-17243). An electronic informed consent was provided before the start of the questionnaire survey, upon completion of the informed consent, study participants filled in the online survey. The study followed all relevant guidelines and regulations.

\section{Consent for publication}

Not applicable

\section{Availability of data and materials}

Data can be made available on request. If you wish to request for the data, please email the corresponding author, Dr. Sobiya Sawani, at sobiya.sawani@aku.edu

\section{Competing interests}

All authors declare that they have no competing interests.

\section{Funding}

The authors have not declared a specific grant for this research from any funding agency in the public, commercial or not-forprofit sectors.

\section{Authors' contributions}

All authors have contributed substantially to this study. SS and ARS conceptualized, designed, and drafted the manuscript of this study. BAU and IA constructed the conduct plan and analytical strategies, reviewed the manuscript several times and provided feedback. AS, HK and SK provided feedback on the proposal development and data collection. All authors have contributed to this manuscript and reviewed and approved the final version of the paper.

\section{Acknowledgements}

Not applicable 


\section{References:}

1. Paul E, Steptoe A, Fancourt D. Attitudes towards vaccines and intention to vaccinate against COVID-19: Implications for public health communications. The Lancet Regional Health-Europe. 2021 Feb 1;1:100012.

2. Krammer F. SARS-CoV-2 vaccines in development. Nature. 2020 Oct;586(7830):516-27.

3. Harapan H, Wagner AL, Yufika A, Winardi W, Anwar S, Gan AK, Setiawan AM, Rajamoorthy Y, Sofyan H, Mudatsir M. Acceptance of a COVID-19 vaccine in Southeast Asia: a cross-sectional study in Indonesia. Frontiers in public health. 2020;8.

4. Ibn-Mohammed T, Mustapha KB, Godsell J, Adamu Z, Babatunde KA, Akintade DD, Acquaye A, Fujii H, Ndiaye MM, Yamoah FA, Koh SC. A critical analysis of the impacts of COVID-19 on the global economy and ecosystems and opportunities for circular economy strategies. Resources, Conservation and Recycling. 2021 Jan 1;164:105169.

5. Harapan H, Itoh N, Yufika A, Winardi W, Keam S, Te H, Megawati D, Hayati Z, Wagner AL, Mudatsir M. Coronavirus disease 2019 (COVID-19): A literature review. Journal of infection and public health. 2020 May 1;13(5):667 - 73.

6. Coronavirus Coverage. (n.d.). National Geographic. Retrieved from https://www.nationalgeographic.com/science/topic/coronavirus-coverage

7. Dzieciolowska S, Hamel D, Gadio S, Dionne M, Gagnon D, Robitaille L, Cook E, Caron I, Talib A, Parkes L, Dubé È. Covid-19 vaccine acceptance, hesitancy, and refusal among Canadian healthcare workers: A multicenter survey. American journal of infection control. 2021 Apr 28.

8. Malik A, Malik J, Ishaq U. Acceptance of COVID-19 vaccine in Pakistan among health care workers. medRxiv. 2021 Jan 1.

9. Hogan A, Winskill P, Watson O, Walker P, Whittaker C, Baguelin M, Haw D, Lochen A, Gaythorpe K, Ainslie K, Bhatt S. Report 33: modelling the allocation and impact of a COVID-19 vaccine.

10. Saad-Roy CM, Wagner CE, Baker RE, Morris SE, Farrar J, Graham AL, Levin SA, Mina MJ, Metcalf CJ, Grenfell BT. Immune life history, vaccination, and the dynamics of SARS-CoV-2 over the next 5 years. Science. 2020 Nov 13;370(6518):811-8.

11. SAGE Working Group on Vaccine Hesitancy Report of the SAGE working group on vaccine hesitancy. Oct 1, 2014. https://www.who.int/immunization/sage/meetings/2014/october/1_Report_WORKING_GROUP_vaccine_hesitancy_final.pdf

12. De Figueiredo A, Simas C, Karafillakis E, Paterson P, Larson HJ. Mapping global trends in vaccine confidence and investigating barriers to vaccine uptake: a large-scale retrospective temporal modelling study. The Lancet. 2020 Sep 26;396(10255):898-908.

13. Fisk RJ. Barriers to vaccination for COVID-19 control-experience from the United States. Global Health Journal. 2021 Feb 9.

14. Majid U, Ahmad M. The factors that promote vaccine hesitancy, rejection, or delay in parents. Qualitative Health Research. 2020 Sep;30(11):1762-76.

15. Sallam M. COVID-19 vaccine hesitancy worldwide: a concise systematic review of vaccine acceptance rates. Vaccines. 2021 Feb;9(2):160.

16. Samo A, Sayed R, Valecha J, Baig N, Laghari Z. Demographic factors associated with acceptance, hesitancy, and refusal of COVID-19 vaccine among residents of Sukkur during lockdown: A cross sectional study from Pakistan. Human Vaccines \& Immunotherapeutics. 2022;:1-5.

17. Ansar F, Naveed H, Khan M, Khattak A. COVID-19 Vaccination hesitancy and associated factors among Pakistani Population. Review of Applied Management and Social Sciences. 2021 Jun 26;4(2):583-94.

18. Yasin A. Covid-19 vaccination target for 2021 met: minister [Internet]. DAWN.COM. 2022 [cited 2 February 2022]. Available from: https://www.dawn.com/news/1666900

19. COVID-19 Health Advisory Platform by Ministry of National Health Services Regulations and Coordination [Internet]. Covid.gov.pk. 2022 [cited 2 February 2022]. Available from: https://covid.gov.pk/

20. Ahmad T, Khan M, Musa TH, Hui J. Polio vaccination campaign in Pakistan: a step towards eradication or still a challenge in hand?. Human vaccines \& immunotherapeutics. 2020 Jun 2;16(6):1444-5.

21. Basharat S, Shaikh BT. Polio immunization in Pakistan: ethical issues and challenges. Public health reviews. 2017 Dec;38(1):1-6. 
22. Arooj S, Ali S, Baber N, Abbasi A, Ali M. Socioeconomic factors effecting polio vaccination in Pakistan.

23. Wang J, Jing R, Lai X, Zhang H, Lyu Y, Knoll MD, Fang H. Acceptance of COVID-19 Vaccination during the COVID-19 Pandemic in China. Vaccines. 2020 Sep;8(3):482.

24. Sallam M, Dababseh D, Eid H, Al-Mahzoum K, Al-Haidar A, Taim D, Yaseen A, Ababneh NA, Bakri FG, Mahafzah A. High rates of COVID-19 vaccine hesitancy and its association with conspiracy beliefs: A study in Jordan and Kuwait among other Arab countries. Vaccines. 2021 Jan;9(1):42.

25. Mercadante AR, Law AV. Will they, or Won't they? Examining patients' vaccine intention for flu and COVID-19 using the Health Belief Model. Research in Social and Administrative Pharmacy. 2021 Sep 1;17(9):1596 - 605.

26. Reno C, Maietti E, Fantini M, Savoia E, Manzoli L, Montalti M et al. Enhancing COVID-19 Vaccines Acceptance: Results from a Survey on Vaccine Hesitancy in Northern Italy. Vaccines. 2021;9(4):378.

27. Harada T, Watanabe T. Changes in Vaccine Hesitancy in Japan across Five Months during the COVID-19 Pandemic and Its Related Factors. Vaccines. 2021;10(1):25.

28. Al-Wutayd O, Khalil R, Rajar AB. Sociodemographic and Behavioral Predictors of COVID-19 Vaccine Hesitancy in Pakistan. Journal of Multidisciplinary Healthcare. 2021;14:2847.

29. Chaudhary FA, Ahmad B, Khalid MD, Fazal A, Javaid MM, Butt DQ. Factors influencing COVID-19 vaccine hesitancy and acceptance among the Pakistani population. Human vaccines \& immunotherapeutics. 2021 Oct 3;17(10):3365-70.

30. Fridman A, Gershon R, Gneezy A. COVID-19 and vaccine hesitancy: A longitudinal study. PloS one. 2021 Apr 16;16(4):e0250123.

31. Wang K, Wong EL, Ho KF, Cheung AW, Yau PS, Dong D, Wong SY, Yeoh EK. Change of willingness to accept COVID-19 vaccine and reasons of vaccine hesitancy of working people at different waves of local epidemic in Hong Kong, China: Repeated cross-sectional surveys. Vaccines. 2021 Jan;9(1):62.

32. Frank K AR. Canadians' willingness to get a COVID-19 vaccine: Group differences and reasons for vaccine hesitancy Statistics Canada. 2020.

33. Qattan A, Alshareef N, Alsharqi O, Al Rahahleh N, Chirwa GC, Al-Hanawi MK. Acceptability of a COVID-19 vaccine among healthcare workers in the Kingdom of Saudi Arabia. Frontiers in Medicine. 2021 Mar 1;8:83.

34. Maraqa B, Nazzal Z, Rabi R, Sarhan N, Al-Shakhra K, Al-Kaila M. COVID-19 vaccine hesitancy among health care workers in Palestine: A call for action. Preventive Medicine. 2021 Aug 1;149:106618.

35. Ning P, Cheng P, Li J, Zheng M, Schwebel DC, Yang Y, Lu P, Mengdi L, Zhang Z, Hu G. COVID-19-Related Rumor Content, Transmission, and Clarification Strategies in China: Descriptive Study. Journal of medical Internet research. 2021 Dec 23;23(12):e27339.

36. Wong LP, Alias H, Wong PF, Lee HY, AbuBakar S. The use of the health belief model to assess predictors of intent to receive the COVID-19 vaccine and willingness to pay. Human vaccines \& immunotherapeutics. 2020 Sep 1;16(9):2204-14.

\section{Figures}




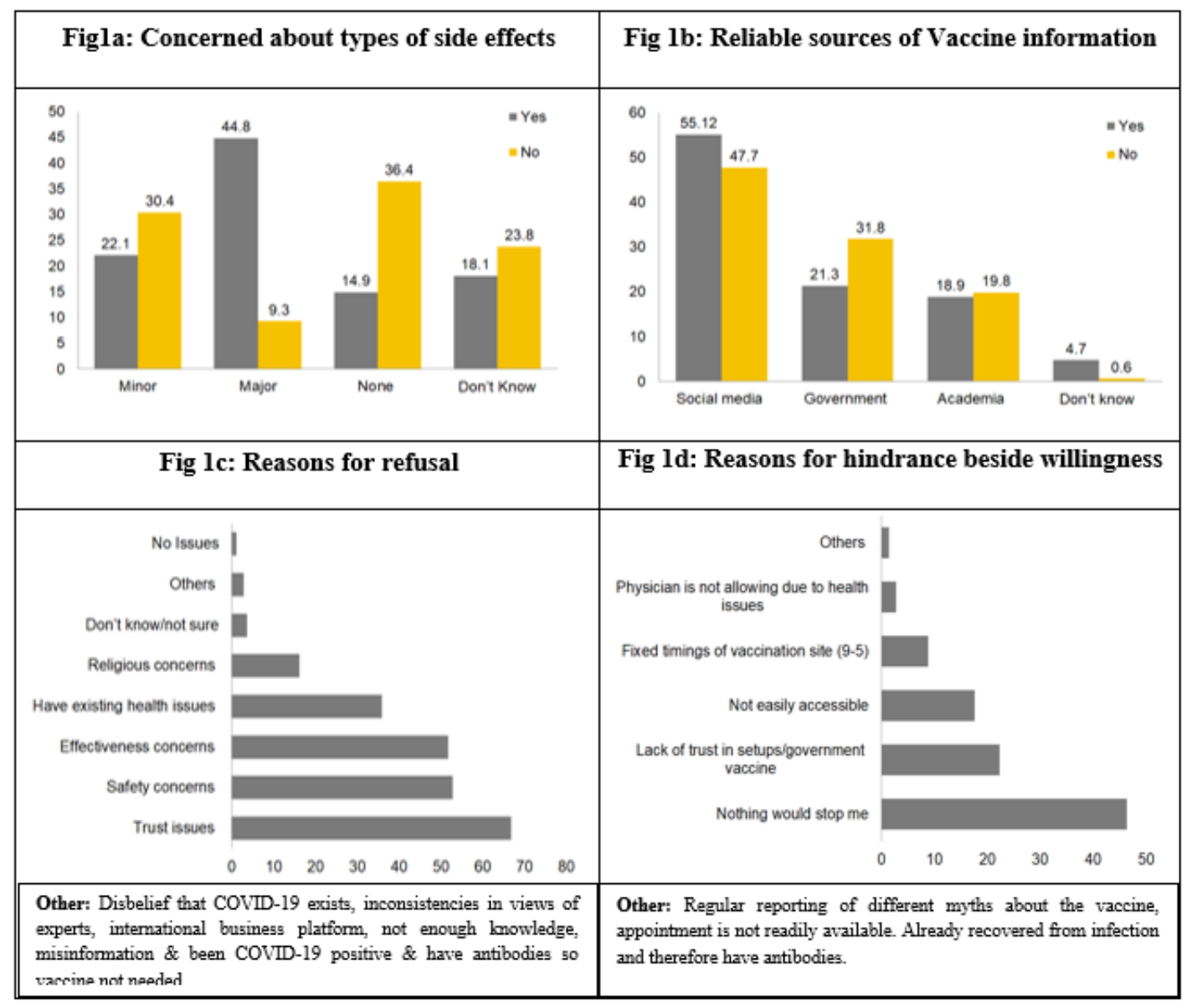

\section{Figure 1}

Vaccine Hesitancy among those (a) who were concerned about types of side effects, (b) reliable sources of COVID-19 vaccine information, (c) reasons for refusal for COVID-19 vaccination \& (d) reasons for hindrance for COVID-19 vaccination besides having willingness 